This is the final peer-reviewed accepted manuscript of:

Shapes, Dynamics and Stability of $\beta$-ionone and Its Two Mutants Evidenced by High-Resolution Spectroscopy.

I. Uriarte, S. Melandri, A. Maris, C. Calabrese, E.J. Cocinero.

The Journal of Physical Chemistry Letters 9 (2018) 1497-1502.

The final published version is available online at:

http://dx.doi.org/10.1021/acs.jpclett.8b00256

Rights / License:

The terms and conditions for the reuse of this version of the manuscript are specified in the publishing policy.

For all terms of use and more information see the publisher's website. 


\title{
Shapes, Dynamics, and Stability of $\beta$-Ionone and Its Two Mutants Evidenced by High-Resolution Spectroscopy in the Gas Phase
}

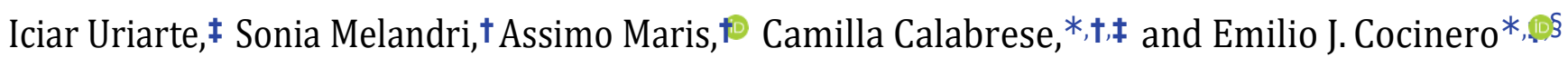 \\ †Dipartimento di Chimica“G. Ciamician”, Universita ' degli Studi di Bologna, via Selmi 2, I-40126 Bologna, Italy

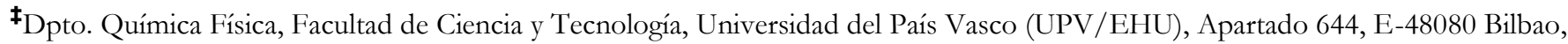 \\ Spain

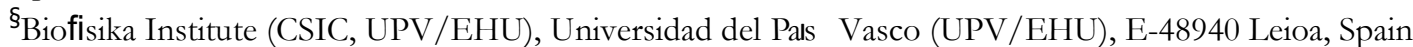

* Supporting Information

ABSTRACT: The conformational landscapes of $\beta$-ionone and two mutants ( $\boldsymbol{\alpha}$-ionone and $\beta$-damascone) have been analyzed by means of state-of-the-art rotational spectroscopy and quantum-chemical calculations. The experiments performed at high resolution and sensitivity have provided a deep insight into their conformational spaces, assigning more than 8000 transitions corresponding to the rotational structures of 54 different species (3 isomers, 14 conformers, and 40 isotopologues). Methyl internal rotation dynamics were also observed and analyzed. The work proved the greatflexibility of $\beta$-ionone due to itsflatter potential energy surface. This feature confers on $\beta$-ionone a wider ability to interconvert between conformers with rather similar energies with respect to its mutants, allowing the retinal ligand to better adapt inside the binding pocket.

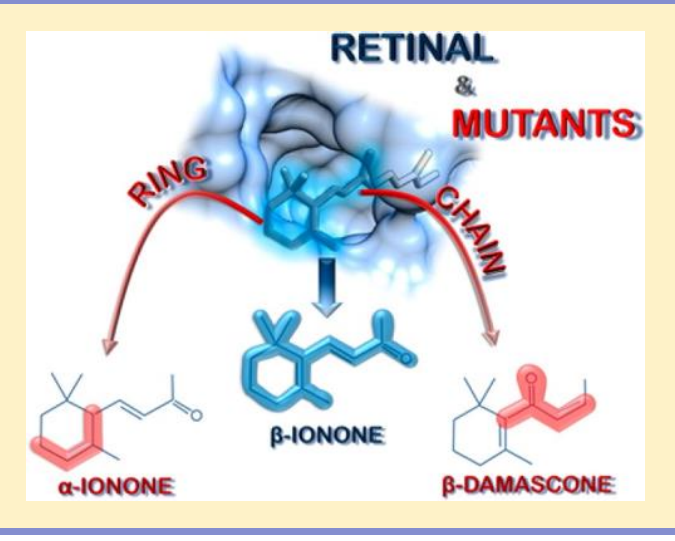

$\mathrm{T}$ the past decade, great interest has been devoted to the 1 conformational analysis of biomolecules. The different factors that govern the stability of peptides, saccharides, nucleic acids, and drugs at a molecular level may be of great importance to the understanding of the mechanisms in which these systems participate in living organisms. ${ }^{1,2}$ Structural studies in solution provide ideal media to compare with the biological

surroundings. On the contrary, gas-phase studies allow for

biomolecules to be probed under isolated conditions, and intrinsic properties of these biosystems can be studied without the interferences of external factors: solvent interactions, crystal packing forces, and so on. Several experimental techniques are available for gas-phase conformational analysis of biomolecules and their clusters. Many of them are based on laser

spectroscopy. ${ }^{2}$ The most precise approach for gas-phase conformational analysis is the use of rotational spectroscopy in combination with supersonic expansions. The inherently

high spectral resolution of this technique allows for the

unambiguous identification of isomers, ${ }^{3}$ conformers, ${ }^{4}$ and isotopologues. ${ }^{5}$ Although initially the technique was restricted to rather small molecular systems, recent advances have revolutionized thefield and have made it possible to study larger systems ${ }^{6,7}$ and also intermolecular complexes. ${ }^{8-10}$ The rich conformational landscape of larger biomolecules (15-25 heavy atoms) characterized by several degrees of freedom is nowadays accessible to rotational spectroscopy. The major recent developments achieved by this technique have been further enhanced by the coupling of laser vaporization to microwaves setups, which avoids decomposition processes of biomolecules, ${ }^{11-14}$ the application of double-quantum coher- ence to phase-discriminate the enantiomeric pair of chiral molecules (three-wave mixing), ${ }^{15}$ and the Chirp Pulse Fourier Transform (CP-FTMW) technique that has revolutionized the field of microwave spectroscopy. ${ }^{16,17}$ Indeed, CP-FTMW instruments allow the recording of broadband rotational spectra in a single acquisition, resulting in shorter spectral acquisition times compared with cavity FTMW experiments. Moreover, millions of rotational spectra can be averaged over time to improve the signal-to-noise ratio.

In this work, the power of the CP-FTMW technique has been exploited recording dense rotational spectra with several thousand lines (more than 8000 lines were assigned), revealing the rotational structure of 54 different species, including isotopologues and methyl internal tunneling states.

This extensive study is aiming at retinal, a very important biochromophore involved in vision processes, which interacts with rhodopsin receptor protein in the form of retinal protonated Schiff-base (RPSB). ${ }^{18}$ This linkage is evident from the position of the UV-Vis absorption maximum, which is strongly affected by a subtle interplay of interactions between the protein and the chromophore. In general, there is common agreement that the interactions between the different protein's amino acid residues and the chromophore induce conformational changes in retinal that vary the wavelength absorption

Received: January 25, 2018

Accepted: March 6, 2018

Published: March 6,2018 
Chart 1. Sketch of the Retinal Structure and Its Analyzed Derivatives, Defining the Corresponding Dihedral Angles ( $\left.\mathbf{T}_{1}, \mathbf{T}_{2}\right)$, and the Atom Numbering Used in the Text ${ }^{a}$

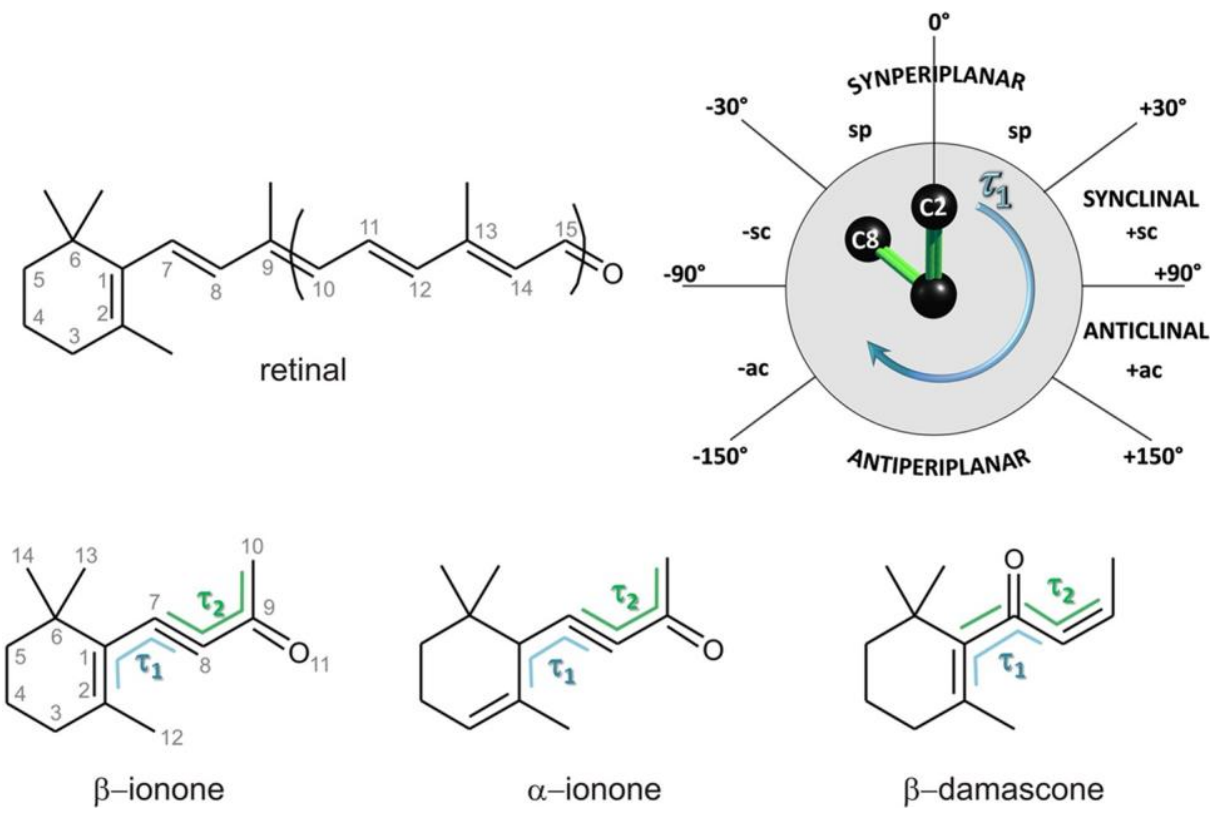

${ }^{a}$ IUPAC nomenclature was used. sp, sc, ac, and ap (synperiplanar $0^{\circ} / \pm 30^{\circ}$, synclinal $30^{\circ} / 90^{\circ}$, anticlinal $90^{\circ} / 150^{\circ}$, and antiperiplanar $\pm 150^{\circ} / 180^{\circ}$ ) forT 1 angle and $\mathrm{C}, \mathrm{T}$ (cis/trans $\left.\sim 0^{\circ} / 180^{\circ}\right)$ forT 2 angle. A conformer labelled as - sc/C would indicate aT $1\left(-30^{\circ}\right.$ and $\left.-90^{\circ}\right)$ andT 2 withcis configuration.

maximum position. ${ }^{19}$ However, the details of these interactions are not clear and are still debated in a variety of studies. ${ }^{20-25}$

Some questions arise. Why has nature settled on this particular motif? Might it present biosynthetic advantages? In this work, we seek information about the structures that act as primary factors in nature's choice of retinal in the visual cycle. The main structural features characterizing thisflexible molecule are a six-membered cyclohexene ring and a lateral chain comprising a double bond and a $\mathrm{C}=\mathrm{O}$ group (see Chart 1). These structural elements have been exhaustively investigated analyzing $\beta$-ionone $(\beta-i)$, considered to be the simplest prototype of retinal, and two "mutants", $\alpha$-ionone $(\alpha-i)$ and $\beta$-damascone $(\beta-d)$, by means of CP-FTMW spectroscopy, supported by theoretical calculations. $\beta$-i (the shorter homologue to the biological unit) gives us the opportunity to explore the conformational landscape, the structure, and the main torsions that govern the retinal biomolecule. The two mutants are useful for evaluating the role of these structural elements in comparison with isomerization on the ring $(\alpha-i)$ and on the side chain $(\beta-d)$. Some previous studies have been performed in the gas phase on ionones, but they did not cover the whole conformational space. $^{26,27}$

It is known that retinal's spectral tuning correlates with the orientation of the alkyl chain with respect to the ring (indicated asT ${ }_{1}$ in Chart 1). In our study, the differentT ${ }_{1}$ arrangements can be explored through the full conformational possibilities of $\beta$-i, while the effects of breaking $\pi$-conjugation are analyzed in $\alpha-i$, where the double bond in the six-membered ring is located in a different position with respect to $\beta-i$, making $C_{1}$ hybridized $\mathrm{sp}^{3}$. The analysis of this feature is interesting because the $\beta-i$ moiety in retinal appears quite free to span the whole torsional space at room temperature. ${ }^{23}$ The interactions of the retinal methyl regions with the receptor residues are also involved in the switching of the spectral tuning process. ${ }^{28}$ Hence, $\beta$ - $d$ was selected to explore the behavior of the terminal methyl group in position 9 and to probe the effect of the closeness of the carbonyl oxygen on the interaction with the ring methyl groups and thus on the structure. The ability to identify these and other more subtle structural differences, particularly those involving the ring and the side chain, provides a striking illustration of the power of rotational spectroscopy.

The conformational landscape of the three related isomers sharing the sum formula $\mathrm{C}_{13} \mathrm{H}_{20} \mathrm{O}$ can be analyzed systematically with respect to the principal torsions that rule their structural arrangements (see Chart 1): $\mathbf{T}_{1}$, the orientation of the alkyl chain with respect to the ring; ${ }_{2}$, the orientation of the double bond of the alkyl chain with respect to the $\mathrm{C}_{10}\left(\mathrm{~T}_{2} \approx 0\right.$ or $\left.180^{\circ}\right)$; and the ring puckering angles, defined by the Cremer-Pople diagram, in which the cyclohexene's minima adopt distorted half-chair configurations (HC, see Figure S4). ${ }^{29}$ These three torsions make possible a characterization of the whole conformational space of each compound.

To predict the most stable conformations adopted by $\beta$ - $i$ and the two mutants, we calculated the potential energy surfaces (PESs) of each molecule by scanning $\mathbf{T}_{1}$ and $\mathbf{T}_{2}$. The ring, due to the double bond, assumes only two HC configurations (twistup and twist-down), with a high inversion barrier close to 20 $\mathrm{kJ} / \mathrm{mol}$ (see Tables S18-S20), in agreement with data reported in literature. ${ }^{30}$ For this reason, the ring was treated as being independent from the side-chain motions. In $\beta-i$ and $\beta-d$, the two positions are equivalent by symmetry $\left({ }^{5} \mathrm{HC}_{4}={ }^{4} \mathrm{HC}_{5}\right.$, where the top and bottom numbers identify the atoms that lie above or below the cyclohexene plane, respectively), whereas for $\alpha-i$, the puckering gives rise to two nonequivalent species $\left({ }^{5} \mathrm{HC}_{6}\right.$ and $\left.{ }^{6} \mathrm{HC}_{5}\right)$, and hence two PESs were calculated. The PESs are depicted in Figure 1 for the three systems as 2D contour plots. Eight different minima are predicted for $\alpha-\mathrm{I}$ (4 ${ }^{5} \mathrm{HC}_{6}$ and $4{ }^{6} \mathrm{HC}_{5}$ ), six for $\beta-i$, and four for $\beta-\mathrm{d}$, the last with a ${ }^{5} \mathrm{HC}_{4}$ ring configuration. The labeling chosen for the 

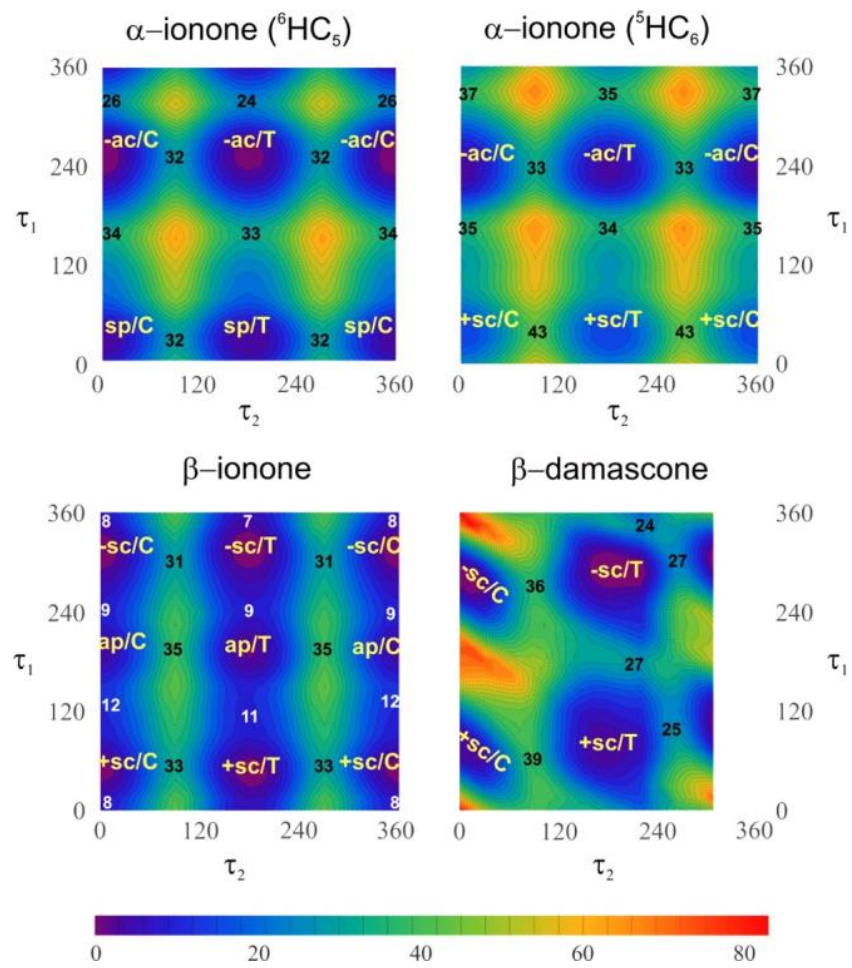

Figure 1.Theoretical 2D conformational PESs $(\mathrm{kJ} / \mathrm{mol})$ of $\alpha$-ionone, $\beta$-ionone, and $\beta$-damascone, exploring theT 1 (ordinate/degrees) and $\mathbf{T}_{2}$ (abscissa/degrees) torsions. The numbers reported on the graphics refer to the barrier heights separating the minima. Noß-damascone data are available for $\mathbf{T}_{2}>315^{\circ}$ because of the steric hindrance between the carbonyl group and the cyclohexene ring. Note that the energy scale of the four PESs is the same, but each molecule has a different absolute minimum energy offset.

identification of the conformers uses the IUPAC nomenclature (see Chart 1).

The rotational spectra $(6-18 \mathrm{GHz})$ of $\beta-\mathrm{i}, \mathbf{\alpha}-\mathrm{i}$, and $\beta-\mathrm{d}$ were collected in three different experiments using the CP-FTMW spectrometer available at the University of the Basque Country. ${ }^{31}$ Experimental details are available in the Supporting Information. The three rotational spectra appear very complex and dense, as shown in Figure 2. In the spectrum ofa-i, six

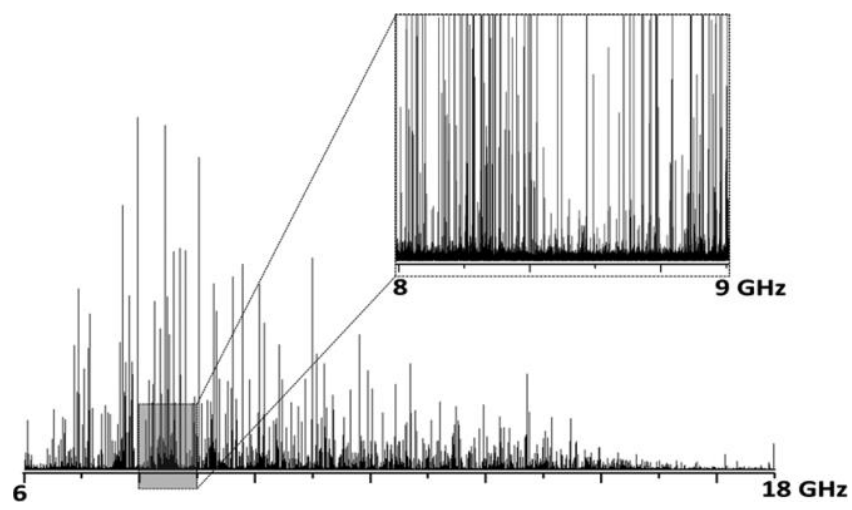

Figure 2.Overview of the CP-FTMW rotational spectrum of $\beta$-ionone. The experimental spectrum is the average of 16.25 million accumulated spectra recorded using three nozzles simultaneously. Signal-to-noise ratio of the most intense assigned line $\left(10_{2,8}-9_{2,7}\right)$ is $\sim 950$. The enlarged portion of the spectrum shows the large number of lines present. different sets of rotational transitions were identified andfit to a semirigid asymmetric rotor Hamiltonian using Watson's Sreduction in the IIIr-representation, ${ }^{32}$ yielding the rotational constants $A, B$, and $C$ reported in Table 1 . Subsequent comparison between experimental and predicted values (see Tables S12-S23) proved that the six observed sets of transitions correspond to conformers ac/ $\mathrm{C}$ and ac/ $\mathrm{T}$ with both ${ }^{5} \mathrm{HC}_{6}$ and ${ }^{6} \mathrm{HC}_{5}$ configurations of the cyclohexene ring and $\mathrm{sp} / \mathrm{C}$ and $\mathrm{sp} / \mathrm{T}$ with a ${ }^{6} \mathrm{HC}_{5}$ configuration of the ring. $\mathrm{A}$ similar procedure was used for $\beta-i$ and $\beta-d$, where four conformers were identified (sc/C, sc/T, sc/C, and sc/T). Besides these 14 structurally different species, a total of $39{ }^{13} \mathrm{C}$ and $1{ }^{18} \mathrm{O}(\mathbf{\alpha}-\mathrm{i})$ isotopologue were also observed and assigned in natural abundance, illustrating the high sensitivity of the employed technique (see the SI for the isotopologues'results.) The experimental rotational constants, reported in Table 1, validate the calculated values, MP2/6-311++G(d,p) and B3LYP-D3/6-311++G(d,p) ${ }^{33}$ (see the complete data set in the SI), showing an excellent agreement. Both methods used are able to characterize the structures with very high precision, giving an average relative error in the rotational constants of $\delta_{\mathrm{MP} 2}=0.15 / 0.42 / 0.81 \%$ and $\delta_{\text {B3LYP-D3 }}=0.48 / 0.40 / 0.35 \%$ for $\beta$ $\mathrm{i}, \boldsymbol{\alpha}-\mathrm{i}$, and $\beta-\mathrm{d}$, respectively.

An experimental estimation of the relative energy between the conformers was obtained following established methods for rotational spectroscopy measurements, ${ }^{34,35}$ assuming that no conformational relaxation occurs during the supersonic expansion. The estimations show that for $\beta-i$, the two lowestlying conformers (sc/C and sc/T) are almost isoenergetic. Moreover, the other conformers (sc/C and sc/T) lie also quite close in energy (2.0(9) and 3.9(9) kJ/mol, respectively). For this reason, at room temperature, the $\beta$-i population is distributed over all of them. The two "mutants"display a slightly different behavior with a locked dominant structure, $-\mathrm{ac} / \mathrm{C}$ for $\alpha-i$ and sc/ $\mathrm{C}$ for $\beta-\mathrm{d}$, and the other conformers lie at much higher energy values. This suggests that for $\beta-i$ the population spans a larger number of conformations than $\alpha-i$ and $\beta$-d, giving greaterflexibility to the system compared with the rigidity of the two mutants (see Tables S1-S3 for the complete relative intensity data).

From the structural point of view, one might think that hyperconjugation in $\beta-i$ would induce planarity of the $C_{2} C_{1}-$ $\mathrm{C}_{7} \mathrm{C}_{8}$ fragment. However, the two conformers that approach planarity (ap/T and ap/C) are predicted to lie higher in energy, and hence they are not observed in our rotational spectra. The steric effects between the hydrogen atom attached to $\mathrm{C}_{8}$ and the hydrogen atoms of the methyl group $\left(\mathrm{C}_{12}\right)$ apparently prevent these arrangements. Thus 1 takes only synclinal orientations $\left(\mathrm{T}_{1} \approx \pm 52^{\circ}\right)$, locking this torsion. However, $\mathbf{T}_{2}$ takes both values $\left(0\right.$ and $\left.180^{\circ}\right)$, and both structures are nearly isoenergetic (see Figure 3). In the mutants, the conformational space changes notably, the most important fact being the observation of a locked dominant structure. In $\beta-d$ the significant difference is in the alkyl chain. Hence, the side chain locks $\mathbf{T}_{2}$ instead of ${ }_{1}$, and it adopts mainly acis configuration with a preference for $\mathrm{a}+\mathrm{sc}$ arrangement of $\mathrm{T}_{1}$ with respect to the cyclohexene ring (see Figure 3). On the contrary, in $\mathbf{a}-\mathrm{i}$ the significant variation is in the ring, obviously causing a change in the puckering of the cyclohexene (see lateranalysis). However, despite having the same side chain as $\beta-i, \alpha-i$ adopts a dominant structure $-\mathrm{ac} / \mathrm{C}$ that was not observed in $\beta$-i (see Figure 3). 
Table 1. Experimental Rotational Constants (in $\mathrm{MHz}$ ) and Experimental Methyl Torsional Barriers (in kJ/mol) of the Observed Conformers

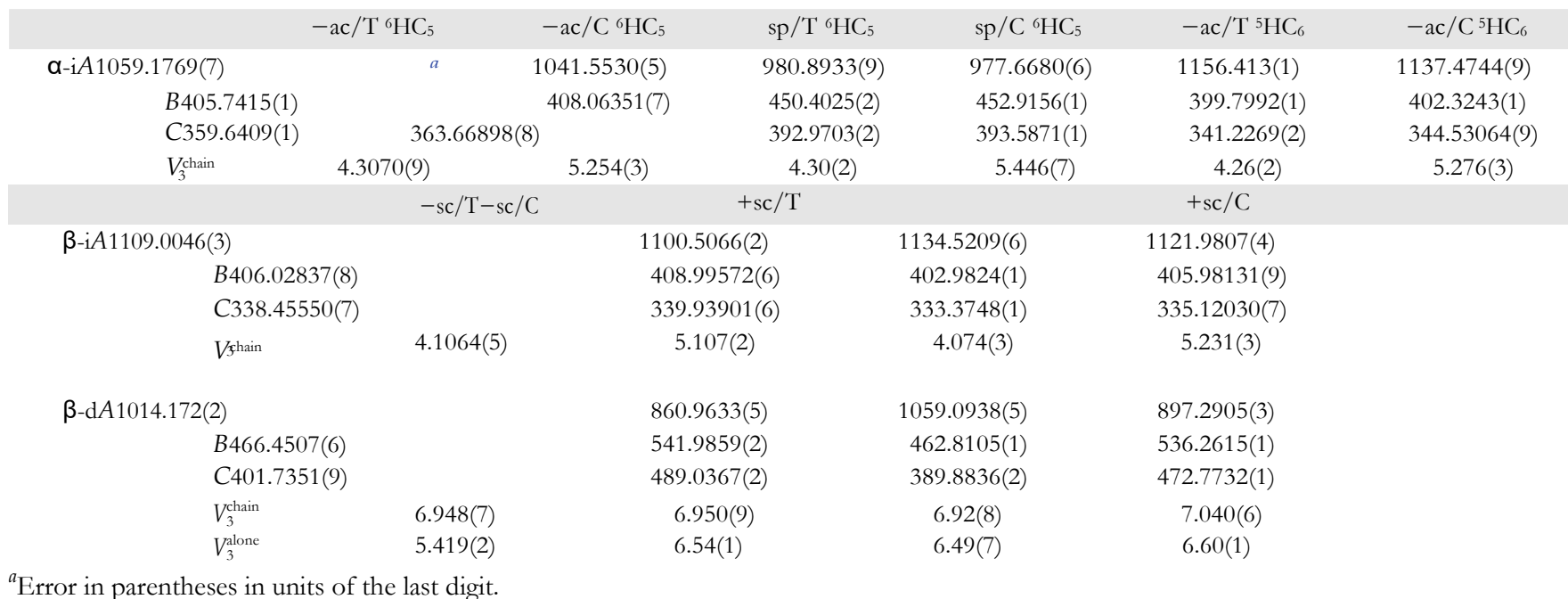

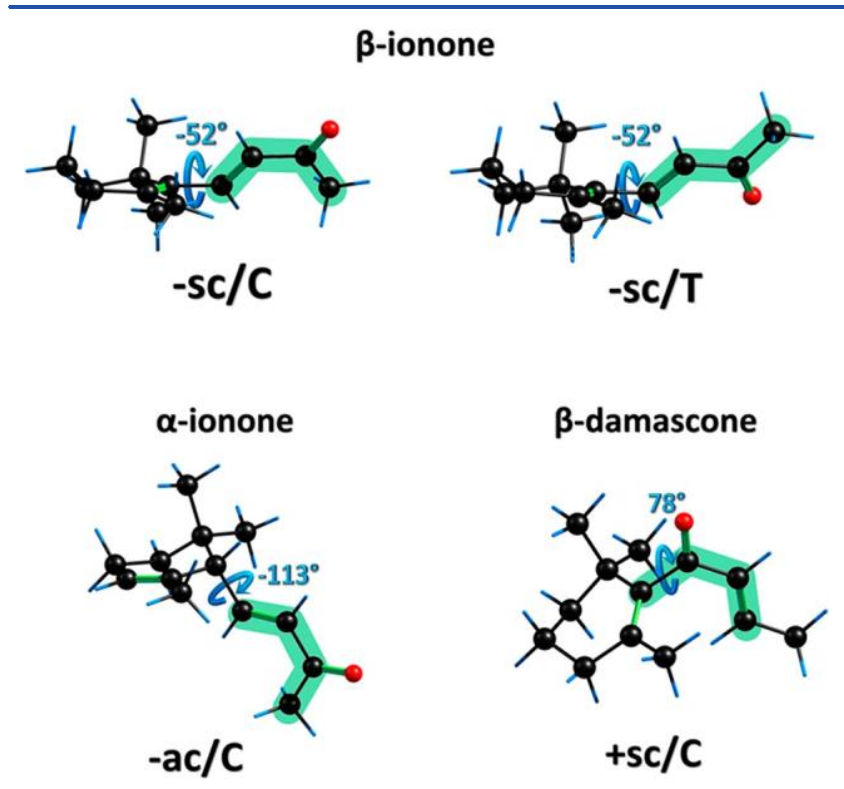

Figure 3.Structures of the observed global minima. $\beta$-ionone exhibits two nearly isoenergetic conformations.

To evaluate the changes in the ring puckering properties among the three molecules, we performed a Cremer-Pople analysis, ${ }^{29}$ which allows the conformational description of a sixmembered ring with three polar coordinates. Each conformation with its corresponding coordinates is located in the spherical visual representation of the Cremer-Pople study in Figure S4. It is clear from the analysis that all three systems share the same structure of the ring $\square$ a distorted HC conformation. Four heavy atoms lie in the same plane, and two heavy atoms stand out of the plane, one atom above and the other below, being on opposite sides of the double bond of the ring. Distinct $\mathrm{HC}$ configurations differ in the identity of the atoms that lie out of the plane. All of the conformations that $\beta-i$ and $\beta-d$ adopt are located in the same part of the sphere, corresponding to a distorted ${ }^{5} \mathrm{HC}_{4}$ conformation, equivalent to ${ }^{4} \mathrm{HC}_{5}$ by symmetry. In $\boldsymbol{\alpha}-\mathrm{i}$, the mutation was introduced in the ring. Hence, $\mathbf{\alpha}-\mathrm{i}$ has a different configuration, the symmetry is lost, and the ring adopts distorted ${ }^{5} \mathrm{HC}_{6}$ and ${ }^{6} \mathrm{HC}_{5}$ conformations.

Besides very precise structural information, rotational spectroscopy can give insights into the internal dynamics in molecules. For the ionones $(\boldsymbol{\alpha}-\mathbf{i}$ and $\beta-i)$, spectroscopic signatures of internal motions related to the chain terminal methyl group were observed, as the rotational lines appeared as doublets. On the contrary, $\beta$-d presented a more complex pattern due to the internal rotations of the chain terminal methyl group and of the methyl attached to $\mathrm{C}_{2}$; here the rotational lines appeared as quintuplets. The observed tunneling splittings lead to an experimental determination of the barriers $\left(V_{3}\right)$ for the methyl torsion. The $V_{3}$ methyl barrier is a probe of the electronic and steric environment of the internal rotor. In the present study, the obtained values (shown in Table 1) could be used for a systematic analysis of the local electronic environment in the different molecules and conformers. In particular, the barrier values of the terminal methyl rotors $\left(V_{3}^{\text {chain }}\right)$ characterize the conformation of the side chain. In fact, the folded carbon chain arrangements present barriers $25 \%$ higher than those of the extended ones $\left(V_{3}^{\text {cis }} \approx 6 \mathrm{~kJ} / \mathrm{mol}\right.$, $V_{3}^{\text {trans }} \approx 4 \mathrm{~kJ} / \mathrm{mol}$; see Table 1$)$, in which the larger distance to the double bond allows for a more free rotation. $\beta$ - $d$ reveals a stand-alone behavior; the two detected methyl torsions give rise to similar $V_{3}$ values for all four minimum energy conformers $(\sim 7 \mathrm{~kJ} / \mathrm{mol})$. The higher $V_{3}$ values of $\beta$-d likely reflect the larger sterical interactions between the carbonyl oxygen and the neighboring methyl regions.

In summary, among the three molecules, $\beta$-i appears to be the mostflexible system with a conformational landscape characterized by barriers low enough to be surmounted under biological conditions (see Figure 1). As population is spread over several conformations, this mobility could be the reason why nature chose $\beta-i$ to be part of the retinal biochromophore. To compare the conformations detected for $\beta-i$ with the structures adopted in the biological environment, a search for the RPSB ligand (RET) was carried out on the Worldwide Protein Data Bank (www.wwpdb.org). ${ }^{36}$ The analyzed structures (5FEN, 5F7G, 5FFH, 5F58, 5F6B, 5FAZ) ${ }^{37}$ present the retinal alkyl chain oriented in a synclinal arrangement, identical to the $\beta$-i conformations observed under isolated conditions (see Figure 4). The elongation of the side chain 


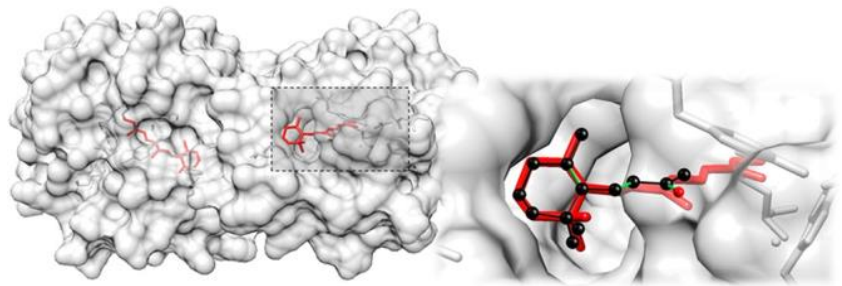

Figure 4.5F6B pdb structure. RET ligand is overlapped with the $-\mathrm{sc} /$ $\mathrm{C} \beta$-i conformation.

makes thetransconfiguration the preferred one, maybe also for maximizing the intermolecular interactions. It can be also noted that in some mutated proteins, ${ }^{19}$ the RET ligand can be found in an antiperiplanar arrangement (4EXZ, 4EEJ, 4EFG, 4GKC; see Table S45), showing the greatflexibility of this moiety to easily overcome the conformational barriers and adopt more fitting structures.

This in-depth gas phase study, supported by comparison between the crystallized and isolated data structures, has revealed that the $\beta$-i moiety is the most appropriate to give to the retinal biochromophore the degrees of freedom required to fulfill its biological function during the photoisomerization process.

\section{ASSOCIATED CONTENT}

\section{Supporting Information}

The Supporting Information is available free of charge on the ACS Publications website atDOI: 10.1021/acs.jpclett.8b00256.

(1) Experimental Section: (1.1) Rotational Spectra (Figures S1-S3); (1.2) Relative Energies (Tables S1S3); (1.3) Experimental versus Ab initio Spectroscopic Parameters (Tables S4-S17). (2) Theoretical Section: (2.1) Cremer-Pople Analysis (Figure S4); (2.2) Ring Inversion Energy Barrier (Tables S18-S20); (2.3) Calculated Spectroscopic Parameters MP2 versus B3LYP-D3 (Tables S21-S23); (2.4) Methyl Internal Rotation Energy Barriers (Tables S24-S26); (2.5) 3D Potential Energy Surfaces (Figure S5); (2.6) Ab initio Geometries (Tables S27-S44). (3) PDB Analysis. (4) Fits of the Observed Conformers and Isotopic Species. (5) References. (PDF)

AUTHOR INFORMATION

\section{Corresponding Authors}

*C.C.: E-mail: calabrese.cami@gmail.com.

*E.J.C.: E-mail: emiliojose.cocinero@ehu.es.

ORCID

Assimo Maris: 0000-0003-2644-0023

Emilio J. Cocinero: 0000-0001-7632-3728

Notes

The authors declare no competingfinancial interest.

\section{ACKNOWLEDGMENTS}

We thank the University of Bologna (RFO2017), the Spanish MINECO (CTQ2017-89150-R), and the UPV/EHU (UFI11/ 23 and PPG17/10) forfinancial support. I.U. and C.C. acknowledge the Spanish Government for an FPU contract and a "Juan de la Cierva" contract, respectively. Computational resources of the UPV/EHU were used in this work (SGIker and I2Basque).

\section{REFERENCES}

(1) Schermann, J.-P.Spectroscopy and Modelling of Biomolecular Building Blocks; Elsevier: Amsterdam, The Netherlands, 2008.

(2) Alonso, J. L.; Boyarkin, O. V.; Carçabal, P.; Cocinero, E. J.; Dunbar, R. C.; Gaigeot, M.-P.; Gloaguen, E.; Lopez, J. C.; Mons, M.; Oomens, J.; et al..Gas-Phase IR Spectroscopy and Structure of Biological Molecules; Rijs, A. M., Oomens, J., Eds.; Springer International Publishing, 2015.

(3) Sanchez, R.; Giuliano, B. M.; Melandri, S.; Favero, L. B.; Caminati, W. Gas-Phase Tautomeric Equilibrium of 4-Hydroxypyrimidine with Its Ketonic Forms: A Free Jet Millimeterwave Spectroscopy Study.J. Am. Chem. Soc.2007,129, 6287-6290.

(4) Vigorito, A.; Calabrese, C.; Paltanin, E.; Melandri, S.; Maris, A. Regarding the Torsional Flexibility of the Dihydrolipoic Acid's Pharmacophore: 1,3-Propanedithiol.Phys. Chem. Chem. Phys.2017, 19, 496-502.

(5) Calabrese, C.; Maris, A.; Uriarte, I.; Cocinero, E. J.; Melandri, S. Effects of Chlorination on the Tautomeric Equilibrium of 2Hydroxypyridine: Experiment and Theory.Chem. - Eur. J.2017,23, 3595-3604.

(6) Alkorta, I.; Cancedda, C.; Cocinero, E. J.; Davalos, J. Z.; Ecija, P.; Elguero, J.; Gonzalez, J.; Lesarri, A.; Ramos, R.; Reviriego, F.; et al. Static and Dynamic Properties of 1,1'-Bi-2-Naphthol and Its Conjugated Acids and Bases.Chem. - Eur. J.2014,20, 14816-14825.

(7) Domingos, S. R.; Cnossen, A.; Buma, W. J.; Browne, W. R.; Feringa, B. L.; Schnell, M. Cold Snapshot of a Molecular Rotary Motor Captured by High-Resolution Rotational Spectroscopy.Angew. Chem., Int. Ed.2017,56,11209-11212.

(8) Calabrese, C.; Gou, Q.; Maris, A.; Caminati, W.; Melandri, S. Probing the Lone Pair $\cdots \pi$-Hole Interaction in Perfluorinated Heteroaromatic Rings: The Rotational Spectrum of Pentafluoropyridine'Water.J. Phys. Chem. Lett.2016,7, 1513-1517.

(9) Perez, C.; Muckle, M. T.; Zaleski, D. P.; Seifert, N. a.; Temelso, B.; Shields, G. C.; Kisiel, Z.; Pate, B. H. Structures of Cage, Prism, and Book Isomers of Water Hexamer from Broadband Rotational Spectroscopy.Science2012,336, 897-901.

(10) Seifert, N. A.; Zaleski, D. P.; Perez, C.; Neill, J. L.; Pate, B. H.; Vallejo-Lopez, M.; Lesarri, A.; Cocinero, E. J.; Castaño, F.; Kleiner, I. Probing the C-H $\cdots$ mWeak Hydrogen Bond in Anesthetic Binding: The Sevoflurane-Benzene Cluster.Angew. Chem., Int. Ed.2014,53, 3210-3213.

(11) Lesarri, A.; Mata, S.; Lopez, J. C.; Alonso, J. L. A Laser-Ablation Molecular-Beam Fourier-Transform Microwave Spectrometer: The Rotational Spectrum of Organic Solids.Rev. Sci. Instrum.2003,74, 4799-4804.

(12) Lesarri, A.; Mata, S.; Cocinero, E. J.; Blanco, S.; López, J. C.; Alonso, J. L. The Structure of Neutral Proline. Angew.Angew. Chem. 2002,114, 4867-4870.

(13) Cocinero, E. J.; Lesarri, A.; Ecija, P.; Basterretxea, F. J.; Grabow, J.-U.; Fernandez, J. A.; Castano, F. Ribose Found in the Gas Phase.

Angew. Chem., Int. Ed.2012,51, 3119-3124.

(14) Lovas, F. J.; Kawashima, Y.; Grabow, J.-U.; Suenram, R. D.; Fraser, G. T.; Hirota, E. Microwave Spectra, Hyperfine Structure, and Electric Dipole Moments for Conformers I and II of Glycine. Astrophys. J.1995,455, L201-L204.

(15) Patterson, D.; Schnell, M.; Doyle, J. M. Enantiomer-Specific Detection of Chiral Molecules via Microwave Spectroscopy.Nature 2013,497, 475-477.

(16) Brown, G. G.; Dian, B. C.; Douglass, K. O.; Geyer, S. M.; Shipman, S. T.; Pate, B. H. A Broadband Fourier Transform Microwave Spectrometer Based on Chirped Pulse Excitation.Rev. Sci. Instrum.2008,79, 053103.

(17) Pate, B. H.; De Lucia, F. C. Broadband Molecular Rotational Spectroscopy Special Issue.J. Mol. Spectrosc.2012,280, 1-2.

(18) Wald, G. The Molecular Basis of Visual Excitation.Science1968, 162, 230-239.

(19) Wang, W.; Nossoni, Z.; Berbasova, T.; Watson, C. T.; Yapici, I.; Lee, K. S. S.; Vasileiou, C.; Geiger, J. H.; Borhan, B. Tuning the 
Electronic Absorption of Protein-Embedded All-Trans-Retinal.Science 2012,338, 1340-1343.

(20) Ferre, N.; Olivucci, M. Probing the Rhodopsin Cavity with Reduced Retinal Models at the CASPT2/CASSCF/AMBER Level of Theory.J. Am. Chem. Soc.2003,125, 6868-6869.

(21) Andersen, L. H.; Nielsen, I. B.; Kristensen, M. B.; El Ghazaly, M. O. A.; Haacke, S.; Nielsen, M. B.; Petersen, M. A. Absorption of SchiffBase Retinal Chromophores in Vacuo.J. Am. Chem. Soc.2005,127, 12347-12350.

(22) Nielsen, M. B. Model Systems for Understanding Absorption Tuning by Opsin Proteins.Chem. Soc. Rev.2009,38, 913-924.

(23) Rajput, J.; Rahbek, D. B.; Andersen, L. H.; Hirshfeld, A.; Sheves, M.; Altoe, P.; Orlandi, G.; Garavelli, M. Probing and Modeling the Absorption of Retinal Protein Chromophores in Vacuo.Angew. Chem., Int. Ed.2010,49, 1790-1793.

(24) Nango, E.; Royant, A.; Kubo, M.; Nakane, T.; Wickstrand, C.; Kimura, T.; Tanaka, T.; Tono, K.; Song, C.; Tanaka, R.; et al. A ThreeDimensional Movie of Structural Changes in Bacteriorhodopsin.

Science2016,354, 1552-1557.

(25) Suomivuori, C. M.; Lang, L.; Sundholm, D.; Gamiz-Hernandez, A. P.; Kaila, V. R. I. Tuning the Protein-Induced Absorption Shifts of Retinal in Engineered Rhodopsin Mimics.Chem. - Eur. J.2016,22, $8254-8261$

(26) Steinmetz, W. E. The Application of Low-Resolution Microwave Spectroscopy to Conformational Analysis.J. Am. Chem. Soc.1974,96, 685-692.

(27) Martin-Drumel, M.-A.; McCarthy, M. C.; Patterson, D.; McGuire, B. A.; Crabtree, K. N. Automated Microwave Double Resonance Spectroscopy: A Tool to Identify and Characterize Chemical Compounds.J. Chem. Phys.2016,144, 124202.

(28) Palczewski, K.; Kumasaka, T.; Hori, T.; Behnke, C. A.; Motoshima, H.; Fox, B. A.; Le Trong, I.; Teller, D. C.; Okada, T.; Stenkamp, R. E.; et al. Crystal Structure of Rhodopsin: A G ProteinCoupled Receptor.Science2000,289, 739 - 745.

(29) Cremer, D.; Pople, J. A. General Definition of Ring Puckering Coordinates.J. Am. Chem. Soc.1975,97, 1354-1358.

(30) Anet, F. A. L.; Freedberg, D. I.; Storer, J. W.; Houk, K. N. On the Potential Energy Surface for Ring Inversion in Cyclohexene and Related Molecules.J. Am. Chem. Soc.1992,114, 10969-10971.

(31) Uriarte, I.; Perez, C.; Caballero-Mancebo, E.; Basterretxea, F. J.; Lesarri, A.; Fernandez, J. A.; Cocinero, E. J. Structural Studies of Nicotinoids: Cotinine versus Nicotine.Chem. - Eur. J.2017,23, 7156.

(32) Watson, J. K. G. InVibrational Spectra and Structure; Durig, J. R., Ed.; Elsevier Science Publishers: New York, 1977.

(33) Grimme, S.; Steinmetz, M. Effects of London Dispersion Correction in Density Functional Theory on the Structures of Organic Molecules in the Gas Phase.Phys. Chem. Chem. Phys.2013,15,

$16031-16042$.

(34) Favero, L. B.; Uriarte, I.; Spada, L.; Écija, P.; Calabrese, C.; Caminati, W.; Cocinero, E. J. Solving the Tautomeric Equilibrium of Purine through Analysis of the Complex Hyperfine Structure of the Four ${ }^{14}$ N Nuclei.J. Phys. Chem. Lett.2016,7, 1187-1191.

(35) Calabrese, C.; Gou, Q.; Maris, A.; Melandri, S.; Caminati, W. Conformational Equilibrium and Internal Dynamics of E-Anethole: A Rotational Study.J. Phys. Chem. B2016,120, 6587-6591.

(36) Rose, P. W.; Prlic, A.; Altunkaya, A.; Bi, C.; Bradley, A. R.; Christie, C. H.; Costanzo, L. D.; Duarte, J. M.; Dutta, S.; Feng, Z.; et al. The RCSB Protein Data Bank: Integrative View of Protein, Gene and 3D Structural Information.Nucleic Acids Res.2016,45, D271D281.

(37) Nossoni, Z.; Nosrati, M.; Geiger, J. H.; Berbasova, T.; Vasileiou, C.; Borhan, B.; Geiger, J. H. Crystal Structure of the Q108K: K40L: T53C Mutant of Human Cellular Retinol Binding Protein II in Complex with All-Trans-Retinal after $24 \mathrm{~h}$ of Incubation at 1.55 Angstrom Resolution. PDB ID: 5FEN, 2016. DOI: 10.2210/pdb5fen/ pdb. 